\title{
Resveratrol ameliorates sepsis-induced acute kidney injury in a pediatric rat model via Nrf2 signaling pathway
}

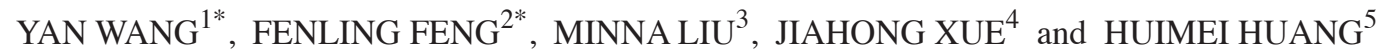 \\ ${ }^{1}$ Department of Pediatrics, Ninth Hospital of Xi'an, Xi'an, Shaanxi 710054; ${ }^{2}$ Department of Pediatrics, \\ Qikang Hospital of Chinese Traditional and Western Medicine, Xi'an, Shaanxi 710000; ${ }^{3}$ Department of Pediatrics, \\ Northwest Women and Children's Hospital, Xi'an, Shaanxi 710061; ${ }^{4}$ Department of Pediatrics, The Affiliated Hospital, \\ Xi'an Jiaotong University; ${ }^{5}$ Department of Pediatrics, Xi'an Children's Hospital, Xi'an, Shaanxi 710000, P.R. China
}

Received October 3, 2016; Accepted June 5, 2017

DOI: $10.3892 /$ etm.2018.6533

\begin{abstract}
Acute kidney injury (AKI) is a hyper-inflammation-induced abrupt loss of kidney function and has become a major public health problem. The cecal ligation and puncture (CLP) model of peritonitis in rat pups mimics the development of sepsis-induced pediatric AKI is pre-renal without morphological changes of the kidneys and high lethality. Resveratrol, a natural polyphenolic compound with low toxicity, has obvious anti-oxidant and anti-inflammatory properties. The present study aimed to determine whether resveratrol alleviates pediatric AKI and investigated the potential mechanism. Thus, a CLP model of 17-18 day-old rat pups was used to mimic the development of sepsis-induced AKI in children. In the group treated with resveratrol, renal injury induced by CLP was alleviated with downregulation of tumor necrosis factor (TNF)- $\alpha$, interleukin (IL)-1 $\beta$ and kidney injury molecule (KIM)-1 expression. Nuclear factor-erythroid-2-related factor 2 (Nrf2) signaling is known to effectively inhibit inflammation, the present study found that resveratrol reduced the lipopolysaccharide-induced inflammatory response in kidney cells in vitro and induced the activation of Nrf2 signaling, including accumulation of nuclear Nrf2 and increase of the expression of Nrf2 target genes heme oxygenase (HO)-1 and NAD(P)H dehydrogenase (quinone) 1 (NQO1); this was confirmed by the induction of the expression of HO-1 and NQO1 by treatment of resveratrol in vitro and in vivo. Of note, knockdown of $\mathrm{Nrf} 2$ effectively abrogated the downregulation of TNF- $\alpha$, IL-1 $\beta$ and KIM-1 expression induced by resveratrol in vitro. These results suggested that resveratrol ameliorates sepsis-induced
\end{abstract}

Correspondence to: Miss Huimei Huang, Department of Pediatrics, Xi'an Children's Hospital, 69 West Juyuan Lane, Xi'an, Shaanxi 710000, P.R. China

E-mail: kakukin01@163.com

\section{${ }^{*}$ Contributed equally}

Key words: AKI, resveratrol, Nrf2, cecal ligation and puncture, pediatric acute kidney injury in a pediatric model of AKI via the Nrf2 signaling pathway.

\section{Introduction}

Sepsis is a condition characterized by a whole-body inflammatory response caused by a harmful host response to infection and is a leading cause of death in children (1). Sepsis is also the second leading cause of acute kidney injury (AKI) in children. AKI is a serious condition defined by the rapid dysfunction of the kidneys within several hours to weeks and has been a serious problem in the pediatric intensive care unit (2). It is worth noting that in the pediatric population, the presence of AKI is associated with an increased mortality compared with that of sepsis alone $(3,4)$. Studies have demonstrated that those patients who survive AKI have a higher risk of future development of chronic kidney diseases (5). However, current therapies for sepsis-induced AKI in pediatric patients are mostly supportive (6). Therefore, it is critical to discover novel and effective therapeutic methods for promoting kidney repair after AKI. Most research on sepsis-induced AKI has utilized adult rodent models; however, the developing kidney in children is more susceptible to oxidative stress (7). The present study used the rat pup cecal ligation and puncture (CLP) model, which displays numerous characteristics of sepsis-induced AKI in pediatric patients with sepsis and has been verified to be suitable for exploring possible therapies (8).

Resveratrol (3,5,4'-trihydroxy-trans-stilbene) is a polyphenolic phytoalexin and is distributed in several food plants such as grapes, berries and peanuts $(9,10)$. Previous studies have revealed that resveratrol exerts protective effects in various disease models, including those of several types of renal injury, via its anti-oxidant and anti-inflammatory properties (11-14). It has been reported that the para-hydroxy group of resveratrol has the ability to scavenge free radicals (15), thus rendering resveratrol with an intrinsic anti-oxidant capacity.

Nuclearfactor-erythroid-2-related factor 2 (Nrf2) is encoded by the NFE2L2 gene and is a basic leucine zipper transcription factor. Under resting conditions, Nrf2 is held in the cytoplasm by its repressor kelch-like ECH-associated protein 1 (Keap1) and removes it through ubiquitination-proteasomal degradation. When cells are exposed to oxidative stress or noxious 
attacks, Nrf2 dissociates from Keap1 and translates to the nucleus, where it binds to the anti-oxidant response element (ARE) within the promoter of its downstream genes, including uridine 5'-diphospho-glucuronosyltransferase, NAD(P) $\mathrm{H}$ :quinone oxidoreductase-1 (NQO1), heme oxygenase-1 (HO-1), glutathione S-transferase, glutamate cysteine ligase and glutathione peroxidase (16). Most of these genes are important anti-oxidant enzymes and cytoprotective genes. It has been reported that $\mathrm{Nrf} 2$-mediated transcriptional responses have an important protective role in ischemic and nephrotoxic acute kidney injury (17). Resveratrol also has protective effects by triggering the Nrf 2 signaling pathway (18-20). However, the protective role of $\mathrm{Nrf} 2$ in sepsis-induced pediatric AKI has remained elusive.

Above all, this evidence led to the hypothesis that resveratrol may alleviate sepsis-induced AKI in a pediatric rat model and induce the activation of Nrf 2 signaling pathway, which was tested in the present study. The results revealed that downregulation of tumor necrosis factor (TNF)- $\alpha$, interleukin (IL)- $1 \beta$ and kidney injury molecule (KIM)-1 expression induced by resveratrol was abrogated in the absence of $\mathrm{Nrf} 2$.

\section{Materials and methods}

Cell culture and transfection. HK2 cells (obtained from the American Type Culture Collection, Manassas, VA, USA) were maintained in Dulbecco's modified Eagle's medium/F12 (Gibco; Thermo Fisher Scientific, Inc., Waltham, MA, USA) supplemented with $10 \%$ fetal bovine serum (Gibco; Thermo Fisher Scientific, Inc.). Cells were cultured in an incubator with a humidified atmosphere of $5 \% \mathrm{CO}_{2}$ in air at $37^{\circ} \mathrm{C}$. Small interfering RNA (siRNA) targeting Nrf2 (siRNA-Nrf2; cat. no. sc-44332) was purchased from Santa Cruz Biotechnology, Inc. (Dallas, TX, USA). An unspecific siRNA with random nucleotides (purchased from Guangzhou RiboBio Co., Ltd., Guangzhou, China) was used as the negative control. Transfection was performed using the Lipofectamine 2000 reagent (Invitrogen; Thermo Fisher Scientific, Inc.).

CLP model. Animal maintenance and experiments were performed in accordance with the guidelines for the care and use of experimental animals of the US National Institutes of Health (Bethesda, MD, USA), and were approved by the Animal Care Committee of Xi'an Children's Hospital (Xi'an, China).

CLP was performed on six male Sprague Dawley rat pups (weight, 40-60 g; purchased from Model Animal Research center Of Nanjing University) aged 17-18 days as described previously (21). All rats were housed under standard conditions (temperature $20-25^{\circ} \mathrm{C}$; humidity $50 \%$ ), received a normal diet and sterile water ad libitum and were exposed to a $12 \mathrm{~h}$ light/dark cycle. The rat pups were anesthetized with $2.5 \%$ isoflurane (Sigma-Aldrich; Merck KGaA, Darmstadt, Germany) and placed on a warm pad. Following laparotomy, $1.5 \mathrm{~cm}$ of the cecal tip was ligated using 4-0 silk and punctured twice with an 18-gauge needle. Approximately $1 \mathrm{~mm}$ of fecal material was expressed from the punctures. In sham-operated pups, the cecum was isolated but neither ligated nor punctured. Following surgery, all rats received $1 \mathrm{ml}$ warm saline via intraperitoneal injection and were placed in individual cages on a heating pad. Rats assessed for durations of $>6 \mathrm{~h}$ were administered intraperitoneal imipenem/cilastatin (14 mg/kg; Sigma-Aldrich; Merck KGaA) and intraperitoneal warm saline $(38 \mathrm{ml} / \mathrm{kg})$ at $6 \mathrm{~h}$ after surgery.

Administration of resveratrol. Fresh resveratrol (Sigma-Aldrich; Merck KGaA) was dissolved in dimethyl sulfoxide and kept in the dark. The CLP rats were intraperitoneally injected with diluted resveratrol $(30 \mathrm{mg} / \mathrm{kg})$ at $6 \mathrm{~h}$ after surgery and then again at $12 \mathrm{~h}$. At $18 \mathrm{~h}$ after CLP, the experimental rats were euthanized. Blood and kidneys were acquired and stored at $-80^{\circ} \mathrm{C}$ for further analysis.

To mimic the AKI in vitro, following the transfection of siRNA, HK2 cells were treated with $10 \mu \mathrm{g} / \mathrm{ml}$ LPS (Sigma-Aldrich; Merck KGaA) for $12 \mathrm{~h}$ at $37^{\circ} \mathrm{C}$, Resveratrol was also administered at the time point.

Plasma electrolyte analysis. Serum creatinine and blood urea nitrogen levels were measured using the QuantiChrom Creatinine/Urea Assay kit (cat. no. DICT-500/DIUR-100; BioAssay Systems, Hayward, CA, USA). Serum IL-1 $\beta$ and TNF- $\alpha$ levels were measured using a MILLIPLEX MAP kit (cat. no. EZRTNFA; EMD Millipore, Billerica, MA, USA).

Reverse transcription-quantitative polymerase chain reaction (RT-qPCR) analysis. Total RNA was isolated from whole kidneys using TRIzol reagent (Invitrogen; Thermo Fisher Scientific, Inc.) according to the manufacturer's instructions. Subsequently, $1 \mu \mathrm{g}$ RNA was converted into complementary (c)DNA using the Transcript first-strand cDNA synthesis kit (cat. no. R111-01/02, Vazyme, Nanjing, China). PCR was performed using SYBR Green PCR Master Mix (Vazyme) and the following primers: GADPH forward, 5'-CCCTCA AGATTGTCAGCAATG and reverse, GTCCTCAGTGTA GCCCAGGAT-3'; IL-1 $\beta$ forward, 5'-CACCTTCTTTTCCTT CATCTTG-3' and reverse, 5'-GTCGTTGCTTGTCTCTCC TTGTA-3'; TNF- $\alpha$ forward, 5'-CATGATCCGAGATGTGGA ACT-3' and reverse, 5'-TCACAGAGCAATGACTCCAAA-3'; KIM-1 forward, 5'-ATGAATCAGATTCAAGTCTTC-3' and reverse, 5'-TCTGGTTTGTGAGTCCATGTG-3'. The thermocycling conditions were as follows: $95^{\circ} \mathrm{C}$ for $5 \mathrm{~min}$; 40 cycles at $95^{\circ} \mathrm{C}$ for $10 \mathrm{sec}$, followed by $60^{\circ} \mathrm{C}$ for $30 \mathrm{sec}$; and $95^{\circ} \mathrm{C}$ for $15 \mathrm{sec}$, followed by $60^{\circ} \mathrm{C}$ for $60 \mathrm{sec}$ and $95^{\circ} \mathrm{C}$ for $15 \mathrm{sec}$. Analysis of the real-time $\mathrm{qPCR}$ data to determine relative gene expression was performed using the $2^{-\Delta \Delta \mathrm{Cq}}$ method (22).

Western blot analysis. Nuclear and cytosolic proteins were isolated using a Nuclear Extraction kit (cat. no. P0027; Beyotime Institute of Biotechnology, Inc., Haimen, China) and the total proteins were acquired from the kidney tissues or cells using cell lysis buffer RIPA (cat. no. P0013B; Beyotime Institute of Biotechnology, Inc.). Protein was quantified using an enhanced BCA protein Assay kit (cat. no. P0009; Beyotime Institute of Biotechnology, Inc.). Proteins $(2 \mu \mathrm{g})$ were separated by $10 \%$ SDS-PAGE and then transferred onto a polyvinylidene difluoride membrane (EMD Millipore). Membranes were then blocked at room temperature using 5\% skimmed milk for $1 \mathrm{~h}$. The following antibodies were used to detect the protein levels: KIM-1 (1:200; cat. no. PA1632; Boster Biological Technology, Wuhan, China), Nrf2 (1:1,000; cat. no. Sc-722; Santa Cruz Biotechnology, Inc.), 
A

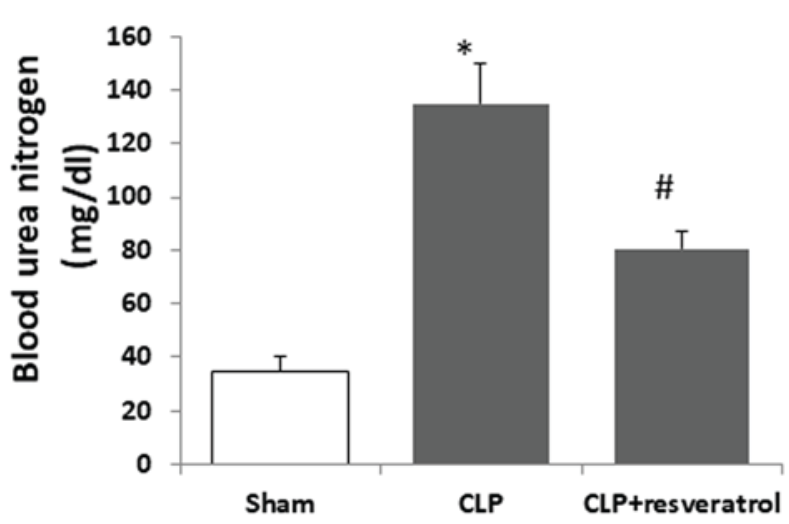

B

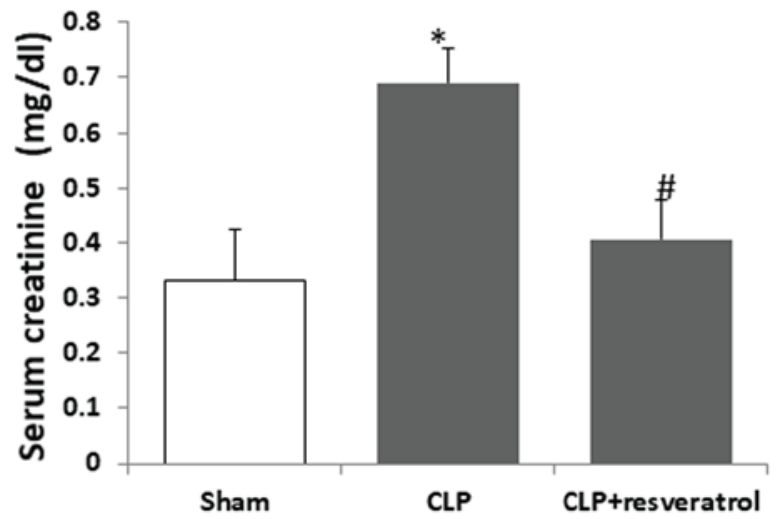

Figure 1. Resveratrol attenuates the decline of renal function induced by CLP. At 18 h post-CLP, (A) blood urea nitrogen and (B) serum creatinine were measured. Values are expressed as the mean \pm standard deviation $(\mathrm{n}=6)$. ${ }^{*} \mathrm{P}<0.05$ vs. Sham; ${ }^{\sharp} \mathrm{P}<0.05$ vs. CLP group. CLP, cecal ligation and puncture.

HO-1 (1:1,000; cat. no. OSA-150C; StressGen, Biotechnologies Corp., Victoria, BC, Canada), NQO1 (1:1,000; cat. no. NB200-209; Novus Biologicals, LLC, Littleton, CO, USA), $\beta$-actin $(1: 5,000$; cat. no. BM0627; Boster Biological Technology), LaminB (1:500; cat. no. sc-6216; Santa Cruz Biotechnology, Inc.), goat anti-rabbit immunoglobulin G (IgG)-horseradish peroxidase (HRP; 1:5,000; cat. no. SN134; Sunshine Bio, Changsha, China) and goat anti-mouse IgG-HRP antibody (1:5,000; cat. no. SN133; Sunshine Bio). Primary antibodies were incubated at $4{ }^{\circ} \mathrm{C}$ overnight, while secondary antibodies were kept at room temperature for $2 \mathrm{~h}$. Results were visualized using an ECL kit (cat. no. E412-01/02; Vazyme, Piscataway, NJ, USA).

Statistical analysis. Values are expressed as the mean \pm standard deviation. Differences between multiple groups were analyzed using one-way analysis of variance, followed by a post-hoc Student-Newman-Keuls test. $\mathrm{P}<0.05$ was considered to indicate a statistically significant difference, SPSS 17.0 software (SPSS, Inc., Chicago, IL, USA) was used to analyze data.

\section{Results}

Resveratrol attenuates the decline of renal function induced by $C L P$. The goal of the present study was to investigate whether resveratrol protects rat pups from renal dysfunction induced by sepsis. A rat pup model of CLP was used to mimic sepsis-induced AKI in children. At the onset of the study, all rat pups were divided into 3 groups $(n=6)$. In the treatment group, rat pups received two injections of resveratrol after CLP surgery.

Serum creatinine and blood urea nitrogen levels are two important measures of kidney function (23). At $18 \mathrm{~h}$ after CLP surgery, serum from the pups in each group was collected for analysis. In CLP model mice, blood urea nitrogen (Fig. 1A) and serum creatinine (Fig. 1B) were significantly elevated compared with those in the sham group. However, resveratrol treatment markedly attenuated the upregulation of urea nitrogen (Fig. 1A) and serum creatinine (Fig. 1B) induced by CLP. These results indicated that resveratrol alleviated the decline of renal function induced by CLP in rat pups.
Resveratrol ameliorates CLP-induced inflammatory response and increase of $K I M-1$. Sepsis is a disseminated inflammatory response and the systemic inflammatory response following CLP is associated with early release of cytokines such as TNF- $\alpha$ and IL-1 $\beta$ (24). CLP not only produces a systemic inflammatory response but also induces inflammation in the kidney. To further verify the effect of resveratrol on sepsis-induced AKI, the levels of TNF- $\alpha$ and IL-1 $\beta$ in serum and kidney tissue were measured in the present study. In the serum, resveratrol significantly inhibited the increase of TNF- $\alpha$ (Fig. 2A) and IL-1 $\beta$ (Fig. 2B) induced by CLP. Similar results were obtained in kidney tissues (Fig. 3A and B).

Kidney injury molecule, a transmembrane type 1 glycoprotein, is a well-characterized biomarker in experimental animals and humans with renal disease (25). The results revealed that KIM-1 mRNA in kidneys was markedly increased after CLP, which was inhibited by resveratrol (Fig. 3C). Resveratrol also attenuated the increase of KIM-1 protein induced by CLP (Fig. 3D).

These results indicated that resveratrol ameliorated CLP-induced AKI in rat pups.

Resveratrol enhances Nrf2 target gene expression in the kidneys. To test whether resveratrol induces Nrf2 function in kidneys during CLP-induced AKI, the Nrf2 target genes HO-1 and NQO1 were evaluated. HO-1 and NQO1 proteins were further increased by resveratrol (Fig. 4A-C). These results indicated that the protective effect of resveratrol on sepsis-induced AKI in a rat pup model was probably due to the activation of $\mathrm{Nrf} 2$.

Resveratrol activates the Nrf2 pathway in vitro. To further verify the effect of resveratrol in Nrf2, HK2 cells were treated with different doses of resveratrol for $3 \mathrm{~h}$. Nrf2 nuclear translocation is a necessary step for Nrf2 activation; therefore, nuclear protein was isolated and assessed for the amount of Nrf2. The results indicated that resveratrol induced the accumulation of Nrf2 in the nucleus (Fig. 5A and B). In this in vitro experiment using HK2 cells, it was also verified that resveratrol increased the expression of HO-1 and NQO1 in a dose-dependent manner 
A

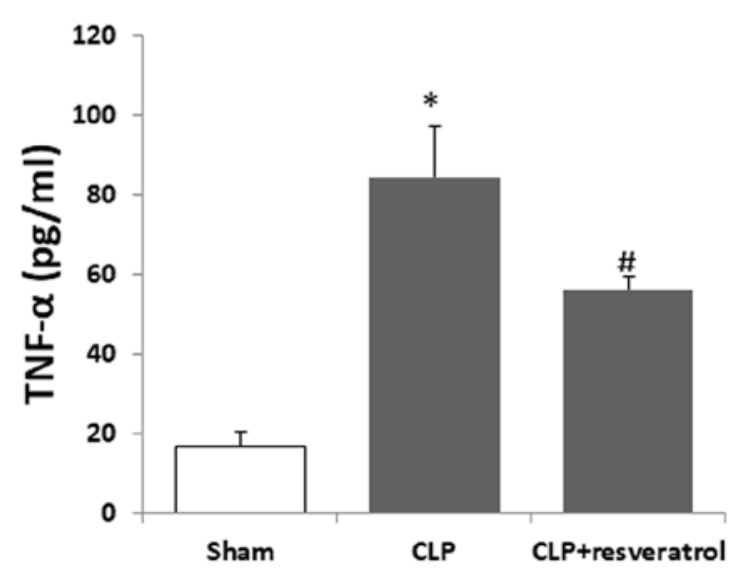

\section{B}

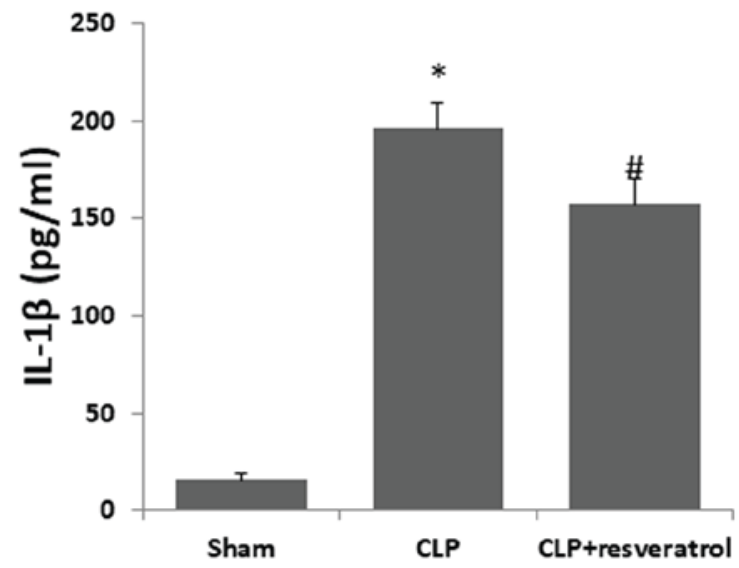

Figure 2. Resveratrol attenuates the CLP-induced increase of serum inflammatory cytokines. Serum levels of the cytokines (A) TNF- $\alpha$ and (B) IL-1 $\beta$ were measured at $18 \mathrm{~h}$ after CLP. Values are expressed as the mean \pm standard deviation $(\mathrm{n}=6)$. ${ }^{*} \mathrm{P}<0.05$ vs. Sham; " $\mathrm{P}<0.05$ vs. CLP group. TNF, tumor necrosis factor; IL, interleukin; CLP, cecal ligation and puncture.

A

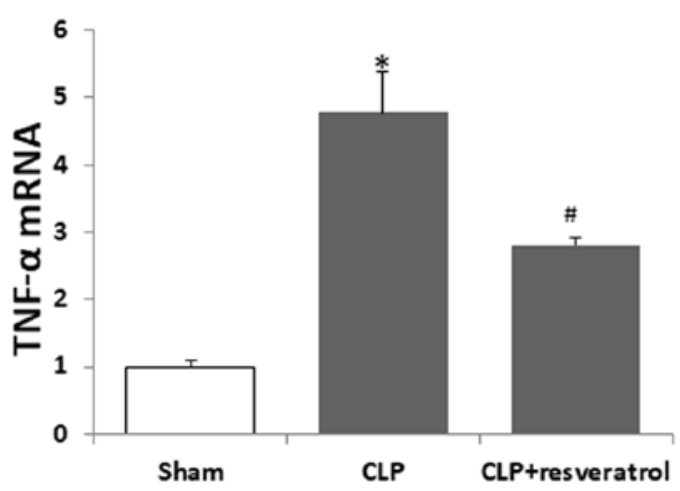

C

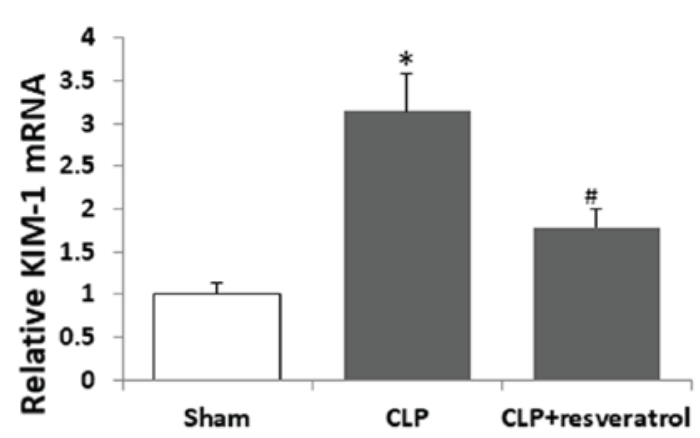

B

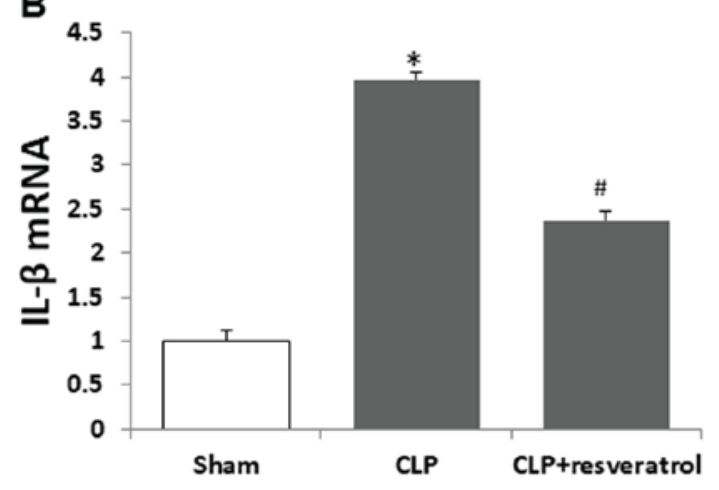

D

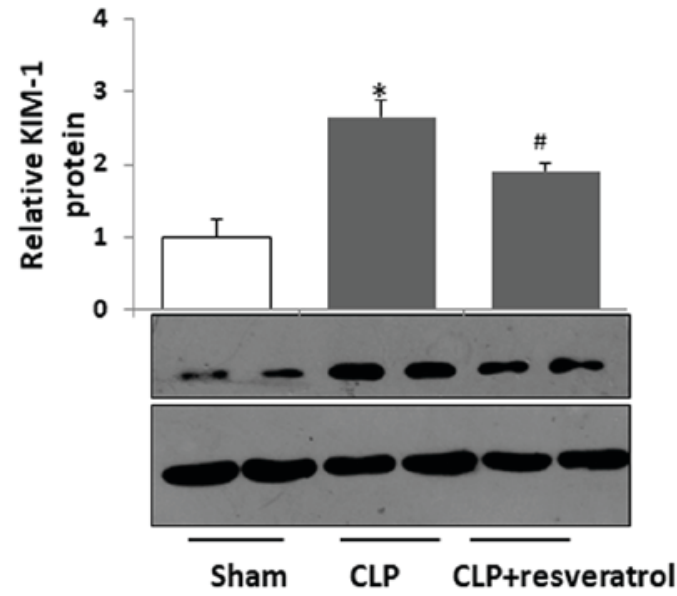

Figure 3. Resveratrol ameliorates CLP-induced increases of inflammatory cytokines and KIM-1 in the kidneys of rat pups. mRNA levels of (A) TNF- $\alpha$, (B) IL-1 $\beta$ and (C) KIM-1 were measured by reverse-transcription quantitative polymerase chain reaction analysis. (D) Protein expression levels of KIM-1 protein were analyzed using western blot analysis. A representative western blot image is presented and quantified expression levels are displayed in the bar graph. Values are expressed as the mean \pm standard deviation $(\mathrm{n}=6)$. " $\mathrm{P}<0.05$ vs. Sham; " $\mathrm{P}<0.05$ vs. CLP group. TNF, tumor necrosis factor; IL, interleukin; CLP, cecal ligation and puncture; KIM-1, kidney injury molecule 1 .

(Fig. 5C and D). Taken together, these results illustrated that resveratrol indeed activated the Nrf2 pathway in HK2 cells.

Knockdown of Nrf2 effectively abrogates the protective effect of resveratrol in vitro. To verify the role of the Nrf2 signaling pathway in the protection of resveratrol, HK2 cells were treated with lipopolysaccharide (LPS) to mimic AKI in vitro. Cells were transfected with siRNA-Nrf2 for $12 \mathrm{~h}$ to knockdown the expression of Nrf2 prior to LPS treatment. Western blot analysis was used to confirm effective knockdown of Nrf2 by 
A

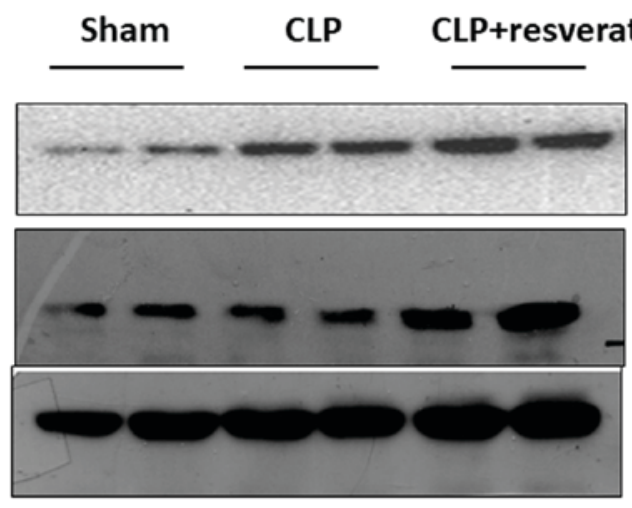

B

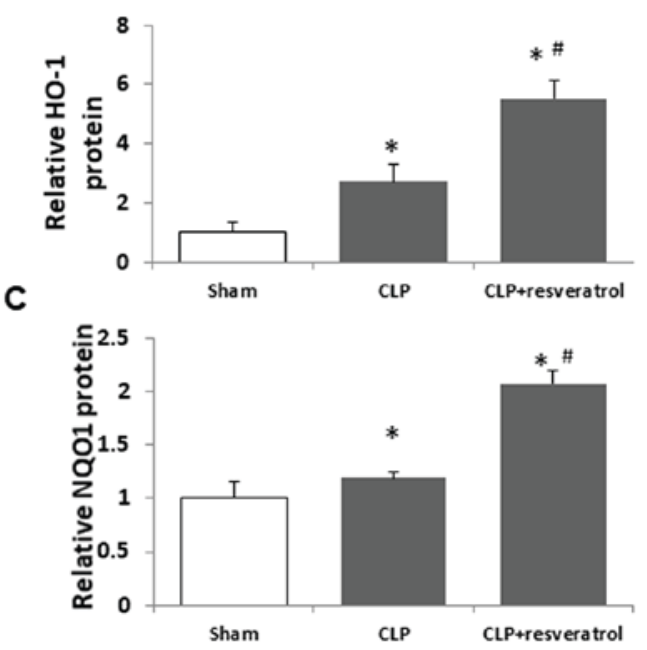

Figure 4. Resveratrol activates nuclear factor-erythroid-2-related factor 2 target gene expression in the kidneys of rat pups with CLP. (A) HO-1 and NQO1 were detected using western blot analysis. Quantified levels of (B) HO-1 protein (C) and NQO1 protein. Values are expressed as the mean \pm standard deviation $(\mathrm{n}=6)$. ${ }^{*} \mathrm{P}<0.05$ vs. Sham group, ${ }^{*} \mathrm{P}<0.05$ vs. CLP group. HO-1, heme oxygenase-1; NQO1, NAD(P)H dehydrogenase (quinone) 1; CLP, cecal ligation and puncture.
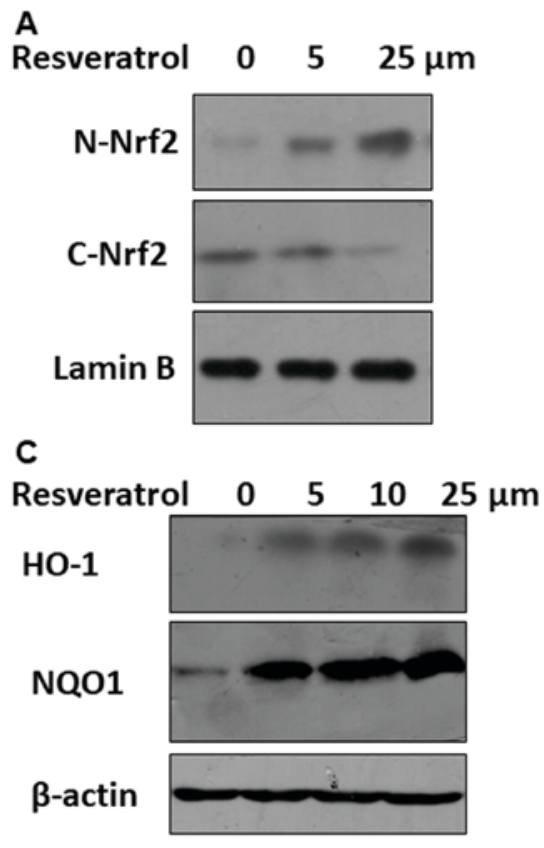
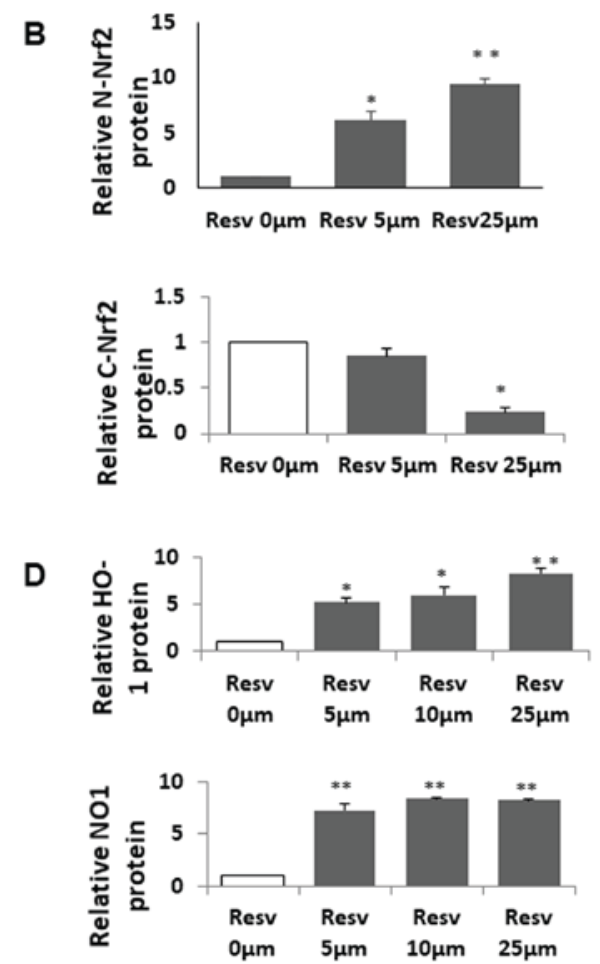

Figure 5. Resveratrol induces the Nrf2 signaling pathway in kidney cells in vitro. (A) Resveratrol induced Nrf2 accumulation in the nucleus. HK2 cells were treated with different doses of resveratrol for $3 \mathrm{~h}$. Nuclear and cytoplasmic proteins were isolated and Nrf2 protein was assessed using western blot analysis. Lamin B protein served as a reference. (B) Quantification analysis of N-Nrf2 (upper panel) and C-Nrf2 (lower panel). (C) Resveratrol increased the expression of Nrf2 target genes. HK2 cells were treated with different doses of resveratrol for $12 \mathrm{~h}$. Total HO-1 and NQO1 protein levels were detected using western blot analysis. (D) Relative protein expression of HO-1 (upper panel) and NQO1 (lower panel) was quantified. The experiments were repeated at least three times, and representative western blots are presented. Values are expressed as the mean \pm standard deviation. ${ }^{*} \mathrm{P}<0.05$ and ${ }^{* * *} \mathrm{P}<0.01$ vs. untreated group. HO-1, heme oxygenase-1; NQO1, NAD(P)H dehydrogenase (quinone) 1; CLP, cecal ligation and puncture; C-Nrf2, cytosolic nuclear factor-erythroid-2-related factor $2 ; \mathrm{N}-$, nuclear.

siRNA-Nrf2 (Fig. 6A). As demonstrated in Fig. 6B, the increase of KIM-1 induced by LPS was ameliorated by resveratrol; however, this effect was abrogated by Nrf2 knockdown. The inflammatory cytokines TNF- $\alpha$ (Fig. 6C) and IL-1 $\beta$ (Fig. 6D) were also assessed. Knockdown of Nrf2 effectively reduced the downregulation of TNF- $\alpha$ and IL- $1 \beta$ expression induced by resveratrol. These results suggested that resveratrol may ameliorate sepsis-induced AKI in a pediatric rat model via the Nrf2 signaling pathway.

\section{Discussion}

Accumulating evidence suggested that resveratrol has numerous beneficial health effects. The present study demonstrated that 

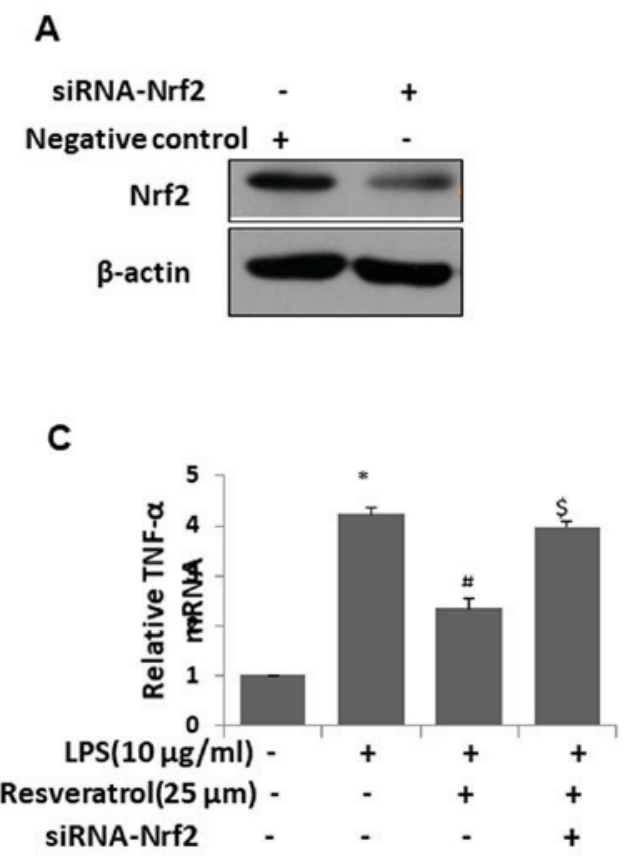
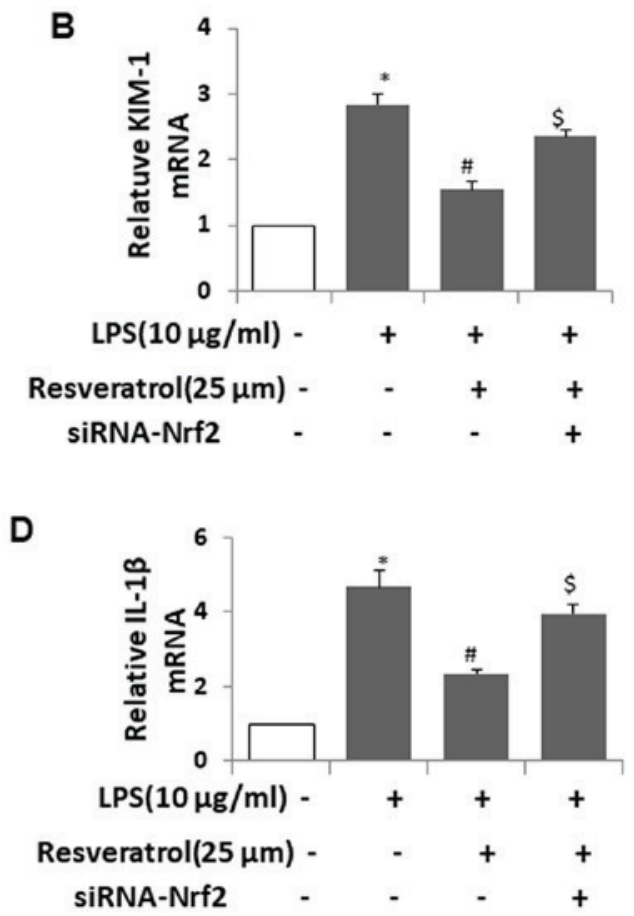

Figure 6. Knockdown of Nrf2 effectively abrogates the protective effect of resveratrol on kidney cells in vitro. (A) HK2 cells were transfected with negative control or siRNA-Nrf2 for $24 \mathrm{~h}$, and the expression of Nrf2 protein was measured by western blot analysis. (B-D) HK2 cells were transfected with negative control or siRNA-Nrf2 for $12 \mathrm{~h}$, and then treated with LPS $(10 \mu \mathrm{g} / \mathrm{ml})$ in the presence or absence of resveratrol for $12 \mathrm{~h}$. RNA was extracted and the mRNA expression of (B) KIM-1 (C) TNF- $\alpha$ and (D) IL-1 $\beta$ was assessed using reverse-transcription quantitative polymerase chain reaction. Values are expressed as the mean \pm standard deviation $(\mathrm{n}=3)$. ${ }^{*} \mathrm{P}<0.05$ vs. control; ${ }^{*} \mathrm{P}<0.05$ vs. LPS group; ${ }^{\$} \mathrm{P}<0.05$ vs. LPS+Resveratol. siRNA, small interfering RNA; TNF, tumor necrosis factor; IL, interleukin; LPS, lipopolysaccharide; Nrf2, nuclear factor-erythroid-2-related factor 2; KIM-1, kidney injury molecule 1.

resveratrol protected against sepsis-induced pediatric AKI in a CLP rat pup model, dependent on the activation of the Nrf2 signaling pathway. In CLP, the Nrf2 signaling pathway was activated and resveratrol was then further activated, which protected against AKI. The inflammatory response and oxidative stress are two important features of sepsis-induced AKI. The Nrf2 signaling pathway not only has a key role in regulating the cellular defense against inflammation but also against oxidative stress and inflammation $(26,27)$. Resveratrol is an Nrf2 signaling pathway activator $(19,28)$. Therefore, the present study investigated resveratrol treatment as a potential therapeutic approach for pediatric sepsis-induced AKI via Nrf2.

Sepsis is a leading cause of death in children worldwide and also an important cause of AKI in pediatric patients (29). The development of AKI significantly incases the mortality of pediatric patients (3). Adult rodent models are generally used for research on sepsis-induced AKI. Of note, the pediatric kidney is still maturing and the developing kidney may be more susceptible to oxidative stress and damage $(7,30)$. It is therefore indicated that the adult animal model may not be the appropriate for studying pediatric AKI. A previous study reported that the rat pup CLP model was able to mimic sepsis-induced AKI in the pediatric population (8). To accurately explore effective therapies of sepsis-induced AKI in children, the present study used this model as a major tool. The rat pup CLP model exhibited elevated serum creatinine and blood urea nitrogen as well as inflammatory factors TNF- $\alpha$ and IL-1 $\beta$, and KIM-1, an important biomarker of AKI. The present study did not detect the morphological changes as in a previous study, which demonstrated that CLP-induced AKI is pre-renal (31). Rat pups injected with resveratrol twice after CLP surgery demonstrated a significantly ameliorated renal function (serum creatinine and blood urea nitrogen) and a reduced inflammatory response and KIM-1 expression compared with those of pups only subjected to CLP treatment. These results verified that resveratrol may be a novel therapy for sepsis-induced AKI in children.

The Nrf2 signaling pathway has a key role in the defense against oxidative stress and inflammatory response. Nrf2 target genes, including HO-1, NQO1 and other phase II-detoxifying enzymes, have significant roles in maintaining cell homeostasis and protecting against tissue injury $(32,33)$. In the present study, resveratrol enhanced the expression of proteins downstream of $\mathrm{Nrf} 2$ in the kidneys of rat pups. In vitro, the HK2 human renal proximal tubule epithelial cells line was utilized to verify the effect of resveratrol on the Nrf2 signaling pathway. Resveratrol induced a rapid translocation of Nrf2 from the cytoplasm to the nucleus and increased the expression of Nrf2 target genes. siRNA was used to knockdown the expression of Nrf2, leading to an inhibition of the effect of resveratrol to attenuate LPS- induced increases of KIM-1, TNF $\alpha$ and IL-1 $\beta$. This evidence verified that resveratrol is an Nrf2 activator in kidney, which may be the underlying mechanism of its renoprotective effect. The Nrf2 target genes HO-1 and phase II-detoxifying enzymes have been reported to regulate the inflammatory response via suppressing nuclear factor (NF)- $\kappa \mathrm{B}$ signaling (16). The Nrf2 signaling pathway suppresses the NF- $\kappa \mathrm{B}$ pathway through decreasing the phosphorylation of the inhibitor of $N F-\kappa B(34)$. Knockout mice (Nrf2-/-) displayed increased $\mathrm{NF}-\kappa \mathrm{B}$ activities and an 
increased susceptibility to LPS-induced sepsis (27). These points illustrated that the Nrf2 signaling pathway is critical to inhibit inflammation. In addition, resveratrol suppressed the expression of TNF- $\alpha$, IL- 6 and cyclooxygenase- 2 through a decrease in the intracellular levels of $\mathrm{Ca}^{2+}$ and extracellular signal-regulated kinase (ERK)1/2 (35). Resveratrol was reported to exert cytoprotective effects through anti-inflammatory, anti-apoptotic and anti-oxidant actions via inducing the activation of sirtuin 1 (36). Resveratrol also alleviates the production of reactive oxygen species (37). These may be the main mechanisms of the protective function of resveratrol in alleviating sepsis-induced AKI. It is worth noting that the importance of Nrf2 in the protective function of resveratrol requires more evidence. It has been illustrated that resveratrol activated Nrf2 through the ERK pathway (38). However, the mechanisms of Nrf2 activation induced by remain to be determined.

In conclusion, the present study illustrated that resveratrol induced Nrf2 activation and alleviated CLP-induced AKI in rat pups. Therefore, resveratrol may have beneficial effects on sepsis-induced AKI in pediatric patients. These results provided a theoretical basis for a novel approach of treating sepsis-induced AKI in pediatric patients using resveratrol. Further study is required to verify the effect of resveratrol in sepsis-induced AKI.

\section{Acknowledgements}

Not applicable.

\section{Funding}

No funding was received.

\section{Availability of data and materials}

The datasets used and/or analyzed during the current study are available from the corresponding author on reasonable request.

\section{Authors' contributions}

$\mathrm{HH}$ conceived and designed the study. JX was a majore contributor in designing the experiments. The rat experiment was mainly completed by YW and the molecular biology experiment by FF. The detection of biochemical indicators was performed by ML and the writing and modification of this article was mainly completed by $\mathrm{JX}$ and $\mathrm{HH}$.

\section{Ethics approval and consent to participate}

The present study was approved by the Animal Care Committee of Xi'an Children's Hospital (Xi'an, China).

\section{Consent for publication}

Not applicable.

\section{Competing interests}

The authors declare that they have no competing interests.

\section{References}

1. Cohen J: The immunopathogenesis of sepsis. Nature 420: 885-891, 2002.

2. Bonventre JV and Yang L: Cellular pathophysiology of ischemic acute kidney injury. J Clin Invest 121: 4210-4221, 2011.

3. Duzova A, Bakkaloglu A, Kalyoncu M, Poyrazoglu H, Delibas A, Ozkaya O, Peru H, Alpay H, Soylemezoglu O, Gur-Guven A, et al: Etiology and outcome of acute kidney injury in children. Pediatr Nephrol 25: 1453-1461, 2010.

4. Fiser RT, West NK, Bush AJ, Sillos EM, Schmidt JE and Tamburro RF: Outcome of severe sepsis in pediatric oncology patients. Pediatr Crit Care Med 6: 531-536, 2005.

5. Chawla LS and Kimmel PL: Acute kidney injury and chronic kidney disease: An integrated clinical syndrome. Kidney Int 82: 516-524, 2012.

6. Brophy PD: Renal supportive therapy for pediatric acute kidney injury in the setting of multiorgan dysfunction syndrome/sepsis. Semin Nephrol 28: 457-469, 2008.

7. Solhaug MJ, Ballèvre LD, Guignard JP, Granger JP and Adelman RD: Nitric oxide in the developing kidney. Pediatr Nephrol 10: 529-539, 1996.

8. Seely KA, Holthoff JH, Burns ST, Wang Z, Thakali KM, Gokden N, Rhee SW and Mayeux PR: Hemodynamic changes in the kidney in a pediatric rat model of sepsis-induced acute kidney injury. Am J Physiol Renal Physiol 301: F209-F217, 2011.

9. Kitada M and Koya D: Renal protective effects of resveratrol. Oxid Med Cell Longev 2013: 568093, 2013.

10. Bertelli AA and Das DK: Grapes, wines, resveratrol, and heart health. J Cardiovasc Pharmacol 54: 468-476, 2009.

11. Sharma S, Anjaneyulu M, Kulkarni SK and Chopra K: Resveratrol, a polyphenolic phytoalexin, attenuates diabetic nephropathy in rats. Pharmacology 76: 69-75, 2006.

12. Palsamy P and Subramanian S: Resveratrol protects diabetic kidney by attenuating hyperglycemia-mediated oxidative stress and renal inflammatory cytokines via Nrf2-Keap1 signaling. Biochim Biophys Acta 1812: 719-731, 2011.

13. Kim MY, Lim JH, Youn HH, Hong YA, Yang KS, Park HS, Chung S, Ko SH, Shin SJ, Choi BS, et al: Resveratrol prevents renal lipotoxicity and inhibits mesangial cell glucotoxicity in a manner dependent on the AMPK-SIRT1-PGC1 $\alpha$ axis in $\mathrm{db} / \mathrm{db}$ mice. Diabetologia 56: 204-217, 2013.

14. Zhang L, Pang S, Deng B, Qian L, Chen J, Zou J, Zheng J, Yang L, Zhang C, Chen X, et al: High glucose induces renal mesangial cell proliferation and fibronectin expression through $\mathrm{JNK} / \mathrm{NF}-\kappa \mathrm{B} / \mathrm{NADPH}$ oxidase/ROS pathway, which is inhibited by resveratrol. Int J Biochem Cell Biol 44: 629-638, 2012.

15. Stojanović S, Sprinz H and Brede O: Efficiency and mechanism of the antioxidant action of trans-resveratrol and its analogues in the radical liposome oxidation. Arch Biochem Biophys 391: 79-89, 2001.

16. Li W, Khor TO, Xu C, Shen G, Jeong WS, Yu S and Kong AN: Activation of Nrf2-antioxidant signaling attenuates NFkappaB-inflammatory response and elicits apoptosis. Biochem Pharmacol 76: 1485-1489, 2008.

17. Liu M, Grigoryev DN, Crow MT, Haas M, Yamamoto M, Reddy SP and Rabb H: Transcription factor Nrf2 is protective during ischemic and nephrotoxic acute kidney injury in mice. Kidney Int 76: 277-285, 2009.

18. Soeur J,Eilstein J,Léreaux G, Jones C and Marrot L: Skin resistance to oxidative stress induced by resveratrol: from $\mathrm{Nrf} 2$ activation to GSH biosynthesis. Free Radic Biol Med 78: 213-223, 2015.

19. Rubiolo JA and Vega FV: Resveratrol protects primary rat hepatocytes against necrosis induced by reactive oxygen species. Biomed Pharmacother 62: 606-612, 2008.

20. Ungvari Z, Bagi Z, Feher A, Recchia FA, Sonntag WE, Pearson K, de Cabo R and Csiszar A: Resveratrol confers endothelial protection via activation of the antioxidant transcription factor Nrf2. Am J Physiol Heart Circ Physiol 299: H18-H24, 2010.

21. Holthoff JH, Wang Z, Seely KA, Gokden N and Mayeux PR: Resveratrol improves renal microcirculation, protects the tubular epithelium, and prolongs survival in a mouse model of sepsis-induced acute kidney injury. Kidney Int 81: 370-378, 2012.

22. Livak KJ and Schmittgen TD: Analysis of relative gene expression data using real-time quantitative PCR and the 2(-Delta Delta C(T)) method. Methods 25: 402-408, 2001.

23. Chen L, Yang S, Zumbrun EE, Guan H, Nagarkatti PS and Nagarkatti M: Resveratrol attenuates lipopolysaccharide-induced acute kidney injury by suppressing inflammation driven by macrophages. Mol Nutr Food Res 59: 853-864, 2015. 
24. Miyaji T, Hu X, Yuen PS, Muramatsu Y, Iyer S, Hewitt SM and Star RA: Ethyl pyruvate decreases sepsis-induced acute renal failure and multiple organ damage in aged mice. Kidney Int 64: $1620-1631,2003$

25. Ichimura T, Bonventre JV, Bailly V, Wei H, Hession CA, Cate RL and Sanicola M: Kidney injury molecule-1 (KIM-1), a putative epithelial cell adhesion molecule containing a novel immunoglobulin domain, is up-regulated in renal cell after injury. J Biol Chem 273: 4135-4142, 1998.

26. Pedruzzi LM, Stockler-Pinto MB, Leite M Jr and Mafra D: Nrf2-keapl system versus NF- $\kappa \mathrm{B}$ : The good and the evil in chronic kidney disease? Biochimie 94: 2461-2466, 2012.

27. Thimmulappa RK, Lee H, Rangasamy T, Reddy SP Yamamoto M, Kensler TW and Biswal S: Nrf2 is a critical regulator of the innate immune response and survival during experimental sepsis. J Clin Invest 116: 984-995, 2006.

28. Soeur J, Eilstein J, Léreaux G, Jones C and Marrot L: Skin resistance to oxidative stress induced by resveratrol: From Nrf2 activation to GSH biosynthesis. Free Radical Biol Med 78: 213-223, 2015.

29. Weiss M, Huber-Lang M, Taenzer M, Kron M, Hay B and Schneider M: Which cut-off should be used in the SOFA score for one organ system to define organ dysfunction, and, thus, severe SIRS/sepsis underlying the 1992 ACCP/SCCM sepsis definitions in critically ill surgical patients? Infection 37: 41-41, 2009.

30. Gupta A, Gupta A, Nigam D, Shukla GS and Agarwal AK Profile of reactive oxygen species generation and antioxidative mechanisms in the maturing rat kidney. J Appl Toxicol 19: 55-59, 1999.

31. Tóth-Heyn P, Drukker A and Guignard JP: The stressed neonatal kidney: From pathophysiology to clinical management of neonatal vasomotor nephropathy. Pediatr Nephrol 14: 227-239, 2000
32. Deng C, Sun ZC, Tong G, Yi W, Ma L, Zhao B, Cheng L, Zhang J, Cao $F$ and Yi D: $\alpha$-lipoic acid reduces infarct size and preserves cardiac function in rat myocardial ischemia/reperfusion injury through activation of PI3K/Akt/Nrf2 pathway. PLoS One 8: e58371, 2013.

33. Zheng H, Whitman SA, Wu W, Wondrak GT, Wong PK, Fang D and Zhang DD: Therapeutic potential of Nrf2 activators in streptozotocin-induced diabetic nephropathy. Diabetes 60: 3055-3066, 2011.

34. Pedruzzi LM, Stockler-Pinto MB, Leite M Jr and Matra D: Nrf2-keap1 system versus NF- $\kappa$ B: The good and the evil in chronic kidney disease? Biochimie 94: 2461-2466, 2012.

35. Csaki C, Mobasheri A and Shakibaei M: Synergistic chondroprotective effects of curcumin and resveratrol in human articular chondrocytes: Inhibition of IL-1beta-induced NF-kappaB-mediated inflammation and apoptosis. Arthritis Res Ther 11: R165, 2009.

36. Kitada M, Kume S, Takeda-Watanabe A, Kanasaki K and Koya D: Sirtuins and renal diseases: Relationship with aging and diabetic nephropathy. Clin Sci (Lond) 124: 153-164, 2013.

37. Huang YT, Chen YY, Lai YH, Cheng CC, Lin TC, Su YS, Liu CH and Lai PC: Resveratrol alleviates the cytotoxicity induced by the radiocontrast agent, ioxitalamate, by reducing the production of reactive oxygen species in HK-2 human renal proximal tubule epithelial cells in vitro. Int J Mol Med 37: 83-91, 2016.

38. Cheng AS, Cheng YH, Chiou $\mathrm{CH}$ and Chang TL: Resveratrol upregulates Nrf2 expression to attenuate methylglyoxal-induced insulin resistance in Hep G2 cells. J Agric Food Chem 60: 9180-9187, 2012 